\title{
Apatinib induces endoplasmic reticulum stress-mediated apoptosis and autophagy and potentiates cell sensitivity to paclitaxel via the IRE-1a-AKT-mTOR pathway in esophageal squamous cell carcinoma
}

Yu-Ming Wang ${ }^{1 \dagger}$, Xin Xu ${ }^{2 \dagger}$, Jian Tang ${ }^{1}$, Zhi-Yong Sun ${ }^{1}$, Yu-Jie Fu' ${ }^{1}$, Xiao-Jing Zhao ${ }^{1}$, Xiu-Mei Ma ${ }^{2 *}$ and Qing Ye ${ }^{1 *}$

\begin{abstract}
Background: Apatinib, a novel vascular endothelial growth factor receptor-2 (VEGFR-2) tyrosine kinase inhibitor, has been approved for the treatment of metastatic gastric cancer and other tumors. Apatinib exerts antiproliferative and proapoptotic effects in different kinds of cancer cells. However, the molecular mechanisms by which apatinib effective against esophageal squamous cell carcinoma (ESCC) have only been partially researched and whether it has a sensitizing effect on paclitaxel remains unclear.
\end{abstract}

Materials and methods: The effects of apatinib or paclitaxel on endoplasmic reticulum (ER) stress, autophagy, apoptosis and proliferation of ESCC cell lines were evaluated. Western blot and immunohistochemistry analyses were performed to detect the expression of related genes. The weight and volume of xenograft tumors in mice were measured.

Results: In the current study, we elucidated the antiproliferative and ER-stress-mediated autophagy-inducing effects of apatinib on ECA-109 and KYSE-150 esophageal squamous cancer cells and identified the underlying mechanisms of its action. We demonstrated that apatinib not only inhibited the proliferation and induced the apoptosis of ESCC cells, but also activated ER stress and triggered protective autophagy. Moreover, inhibiting autophagy by chloroquine (CQ) enhanced the apatinib-induced apoptosis of ESCC cells through the IRE-1 a-AKT-mTOR pathway. In addition, we showed, for the first time, the paclitaxel combined with apatinib and CQ exhibited the best antitumor effect on ESCC both in vivo and in vitro via the IRE-1a-AKT-mTOR pathway.

Conclusions: Our data showed that apatinib induced ER stress, autophagy and apoptosis in ESCC. Inhibiting autophagy by CQ enhanced apatinib-induced apoptosis. The combination of apatinib and CQ sensitized ESCC cells to

*Correspondence: sallyma@hotmail.com; renjiyeqing@163.com

†Yu-Ming Wang and Xin Xu contributed equally to this work

${ }^{1}$ Department of Thoracic Surgery, Renji Hospital, School of Medicine,

Shanghai Jiao Tong University, Shanghai 200127, People's Republic

of China

2 Department of Radiation Oncology, Renji Hospital, School of Medicine,

Shanghai Jiao Tong University, Shanghai 200127, People's Republic

of China

(c) The Author(s) 2021. This article is licensed under a Creative Commons Attribution 4.0 International License, which permits use, sharing, adaptation, distribution and reproduction in any medium or format, as long as you give appropriate credit to the original author(s) and the source, provide a link to the Creative Commons licence, and indicate if changes were made. The images or other third party material in this article are included in the article's Creative Commons licence, unless indicated otherwise in a credit line to the material. If material is not included in the article's Creative Commons licence and your intended use is not permitted by statutory regulation or exceeds the permitted use, you will need to obtain permission directly from the copyright holder. To view a copy of this licence, visit http://creativeco mmons.org/licenses/by/4.0/. The Creative Commons Public Domain Dedication waiver (http://creativecommons.org/publicdomain/ zero/1.0/) applies to the data made available in this article, unless otherwise stated in a credit line to the data. 
paclitaxel to induce apoptosis through the IRE-1a-AKT-mTOR signaling pathway, thus providing the basis for its use in innovative anticancer therapeutic strategies.

Keywords: Apatinib, ER stress, Autophagy, Apoptosis, Paclitaxel, Esophageal squamous cell carcinoma

\section{Introduction}

ESCC is the type of esophageal cancer predominant in East Asia, especially in China, where it accounts for more than $90 \%$ of cases $[1,2]$. It has been reported that there are more than 280,000 new cases of ESCC every year in China [3-6]. Despite advances in diagnosis and therapy in recent decades, the 5-year survival rate remains low at an estimated 15-20\% [7].

Surgery is the primary treatment for early-stage ESCC; however, more than one-half of patients are found to have locally advanced cancer at diagnosis, and therefore, they are offered neoadjuvant or adjuvant therapy. The combination of platinum-based 5 -fluorouracil and paclitaxel is currently the most frequently used perioperative chemotherapy [8]. The combination of chemotherapy and targeted therapy has provided opportunities for improving outcomes in recent years. However, only 3 targeted therapeutic agents including trastuzumab, ramucirumab, and pembrolizumab, have been approved by the FDA for use in esophageal and EGJ cancers [9]. Most researches about targeted therapy are still in the clinical or basic research stage. Apatinib, as a novel VEGFR-2 tyrosine kinase inhibitor [10, 11], is the second antiangiogenic drug that has been approved for the therapy of advanced metastatic gastric cancer [12]. In addition to gastric cancer, apatinib also plays an important role in mitigating other solid tumors, including thyroid cancer, gynecologic cancer, hepatocellular carcinoma (HCC) and esophageal cancer [13-16]. There are relatively few studies of apatinib with respect to esophageal cancer, and the application value and corresponding mechanism of apatinib when combined with chemotherapy are not sufficiently clear [13, 17-19].

The endoplasmic reticulum is a dynamic organelle with significant functions including folding of newly synthesized proteins and maintenance of calcium homeostasis [20, 21]. ER stress occurs when homeostatic processes are disrupted and results in the accumulation of unfolded or misfolded proteins [22]. To resist ER stress and maintain cellular homeostasis, autophagy is triggered to degrade and recycle cytoplasmic materials and thus has mostly a pro-survival function in cancer cells. Moreover, autophagy and apoptosis are closely related. Generally, autophagy prevents the induction of apoptosis through the inactivation of caspases. However, under special circumstances, autophagy or autophagy-related proteins might facilitate apoptosis induction. These findings indicate that the duality of autophagy might be related to the regulation of apoptosis of cells under ER stress $[23,24]$.

In this study, we aimed to clarify the potential therapeutic effects and relevant molecular mechanisms of apatinib in ESCC in vivo and in vitro and to explore the sensitizing effect of apatinib on cells treated with paclitaxel. We first found that apatinib had an effective antitumor effect by inhibiting tumor proliferation, invasion and migration in ESCC. Then, we found that apatinib induced ER stress, autophagy and apoptosis. Inhibiting autophagy by CQ administration significantly enhanced anticancer effects of apatinib via the IRE- $1 \alpha-A K T-m T O R$ signaling pathway. Finally, we explored whether the combination of apatinib and CQ could sensitize ESCC cells to paclitaxel to induce apoptosis both in vivo and in vitro.

\section{Materials and methods \\ Cell cultures}

ECA-109 cells (RRID: CVCL_6898) and KYSE-150 cells (RRID: CVCL_1348) (Stem Cell Bank, Shanghai, China) were authenticated using STR (or SNP) profiling within the last three years and were cultured in RPMI-1640 or DMEM (Sigma, St. Louis, MO, USA) with $10 \%$ FBS (Gibco, USA), $100 \mathrm{U} / \mathrm{ml}$ streptomycin and $100 \mathrm{U} / \mathrm{ml}$ penicillin (Millipore, TMS-AB2-C) at $37{ }^{\circ} \mathrm{C}$ in a humidified incubator with $5 \% \mathrm{CO}_{2}$. All experiments were performed with mycoplasma-free cells. To detect autophagic flux, the ECA-109 and KYSE-150 cells were transfected with RFP-GFP-hLC3 
lentivirus (Jiman, Shanghai); the specific procedures were described previously [25].

\section{Reagents and antibodies}

Apatinib was obtained from Hengrui Medicine Co., Ltd. (Jiangsu, China). Paclitaxel, CQ, SC79, rapamycin and bafilomycin A1 were purchased from Selleck Chemicals (Houston, TX, USA). Paclitaxel was purchased at a concentration of $10 \mathrm{mM}$, which was soluble in DMSO. Serum-free medium was used to dilute the drug to working concentration $(10 \mu \mathrm{M})$ and the $\mathrm{v} / \mathrm{v} \%$ was $0.1 \%(1: 1000)$. Apatinib was purchased in the form of powder. $10 \mathrm{mg}$ apatinib was dissolved in $25.159 \mu \mathrm{l}$ DMSO to get the drug with a concentration of $1 \mathrm{M}$. Then, the serum-free medium was used to dilute the drug to working concentration $(25 \mathrm{mM})$ and the $\mathrm{v} / \mathrm{v} \%$ was $2.5 \%$ (1:40).

Antibodies including anti-Bax (89477), anti-Bcl2 (15071), anti-Caspase3 (14220), anti-Cleaved-Caspase3 (9664), anti-PARP (9532), anti-Cleaved-PARP (5625), anti-Chop (2895), anti-GRP78 (3177), antiIRE-1 $\alpha$ (3294), anti-JNK (9252), anti-Beclin1 (3495), anti-LC3 (12741), anti-P62 (23214), anti-AKT (4685), anti-pAKT (4060), anti-mTOR (2983), anti-pmTOR (5536), anti-GAPDH (2118) were all purchased from Cell Signaling Technology (Beverly, MA, USA).

\section{Cell viability assays}

A Cell Counting Kit-8 (Dojindo, Kumamoto, Japan) was used to assess the cytotoxicity of drugs on ESCC cell lines. Approximately 2500 cells were plated in 96-well plates $24 \mathrm{~h}$ before they were treated with different drugs. The absorbance at $450 \mathrm{~nm}$ was detected by a microplate reader.

\section{Colony formation assay}

A total of 1000 cells were seeded in 6-well plates and incubated for 2 weeks after treatment with drugs and then stained with $1 \%$ crystal violet before being photographed and quantified.

\section{Apoptosis rate and cell cycle analyses}

Cells were seeded in six-well plates for $24 \mathrm{~h}$ and then treated with apatinib or paclitaxel for another $24 \mathrm{~h}$. The harvested cells were washed twice with cold PBS and treated with $5 \mu \mathrm{l}$ of propidium iodide (PI, $50 \mu \mathrm{g} /$ $\mathrm{ml}$ ) and $3 \mu \mathrm{l}$ of FITC-Annexin $\mathrm{V}$ for $15 \mathrm{~min}$ at room temperature in the dark. Three hundred microlitres of 1xbinding buffer were added to each sample. The number of apoptotic cells was determined with a FACS-Caliber system (BD Biosciences, USA). For cell cycle analysis, the preparation of cells was similar to that described for the apoptosis analysis and were fixed overnight in $70 \%$ ethanol at $4{ }^{\circ} \mathrm{C}$. Then, the cells were washed twice with cold PBS and incubated with PI $(50 \mu \mathrm{g} / \mathrm{ml})$ for one-half hour in the dark. Finally, all samples were analyzed by a FACS Caliber system. In the above experiment, 3 independent events were set for each group, and the results were averaged.

\section{Western blot analysis}

Protein samples from treated cells were extracted and quantified by a BCA protein assay kit (Pierce, Rockford, USA), subjected to SDS-PAGE and transferred to PVDF membranes (Millipore, Temecula, California, USA). Then, the bolts were probed with primary and secondary antibodies.

\section{Confocal microscopy}

We used 4\% paraformaldehyde (Sigma) to fix cells for $20 \mathrm{~min}$ and $0.1 \%$ Triton X-100 (Sigma) to permeabilize cells for $15 \mathrm{~min}$ at room temperature. Then, the cells were washed with PBS and blocked with PBS containing $0.5 \%$ bovine serum albumin (BSA) and $0.15 \%$ glycine (BSA buffer) for $1 \mathrm{~h}$ at room temperature. Finally, the slices were treated with DAPI (Sigma) and imaged by confocal microscopy (Zeiss).

\section{Intracellular $\mathrm{Ca}^{2+}$ detection}

We used Fluo3 AM (S1056, Beyotime, Shanghai, China) to detect intracellular $\mathrm{Ca}^{2+}$ levels. Flow cytometry was used to detect the fluorescence intensities of Fluo3 signals and determine $\mathrm{Ca}^{2+}$ binding.

\section{Electron microscopy}

After treatment, cells were fixed in $2 \%$ glutaraldehyde and $2 \%$ paraformaldehyde in $0.1 \mathrm{~mol} / \mathrm{l}$ sodium phosphate buffer ( $\mathrm{pH} 7.4)$ at $4{ }^{\circ} \mathrm{C}$ for $3 \mathrm{~h}$. After postfixing in $1 \%$ osmium tetroxide in the same buffer for $2 \mathrm{~h}$ and gradual dehydrated in an alcohol gradient, the samples were embedded in a mixture of EPON 618 and epoxypropane. Ultrathin sections were stained with $5 \%$ uranyl acetate and Reynold's lead citrate and semithin sections were stained with toluidine blue. An 
electron microscope equipped with a digital camera was used to examine the sections.

\section{RNA interference}

Cells were treated with IRE- $1 \alpha$ or Beclin1 siRNA (200 nM, BioeGene, Shanghai, China). The siRNA sequences of IRE- $1 \alpha$ were as follows: siRNA-1 5'-CCA AGAUGCUGGAGAGAUUTT- ${ }^{\prime}$ (sense); 5'-AAU CUCUCCAGCAUCUUGGTA-3' (antisense). siRNA-2 5'-GGAAGUUAUCAACCUGGUUTT-3' (sense); 5'-AACCAGGUUGAUAACUUCCTG-3' (antisense). SiRNA-3 5'-GCAGAAGGACUUUGCGCAUTT-3' (sense); 5'-AUGCGCAAAGUCCUUCUGCTC-3' (antisense). The siRNA sequences of Beclin1 were as follows: siRNA-1 5'-GCUCAGUAUCAGAGAGAA UTT-3' (sense); 5'-AUUCUCUCUGAUACUGAG CTT-3' (antisense). siRNA-2 5'-GGUCUAAGACGU CCAACAATT-3' (sense); 5'-UUGUUGGACGUC UUAGACCCT-3' (antisense). siRNA-3 5'-CCCAGG AGGAAGAGACUAATT-3' (sense); 5'-UUAGUCUCU UCCUCCUGGGTC-3' (antisense). Western blotting was used to detect the effect of IRE- $1 \alpha$ or Beclin 1 siRNAs, and select the siRNA with the best knockout effect for follow-up experiments (Additional file 1: Figure $\mathrm{S} 1 \mathrm{a}-\mathrm{f}$ ).

\section{Xenograft tumor model, immunochemistry and TUNEL assay}

We purchased four-week male $\mathrm{BALB} / \mathrm{c}$ nude mice from the Institute of Zoology, Chinese Academy of Sciences of Shanghai and carried out all the experiments strictly according to the Guide for the Care and Use of Laboratory Animals [26]. Research was approved by the Institutional Animal Care and Use Committee at the Shanghai Jiaotong University School of Medicine (Approval ID: A-2018-024). ECA109 cells $\left(1 \times 10^{6}\right.$ cells in $100 \mu$ of PBS per mouse $)$ were subcutaneously injected into the right flanks of the nude mice by. After 14 days, all animals were randomly assigned to 6 groups, which were shown in Table 1. Tumor volume was calculated on the basis of weekly measurement using the following formula: $\mathrm{V}=0.5 \times$ length $\mathrm{x}$ width ${ }^{2}$. All mice were sacrificed 6 weeks after ECA-109 cell inoculation, and xenograft tumors were weighed and fixed for immunohistochemistry (IHC) staining. A TUNEL assay was carried out to determine the apoptotic cells using an in situ cell death detection kit with Fluorescein (Roche Applied Science, USA).

\section{Statistical analyses}

All data were analyzed using GraphPad 6.0 and SPSS 18.0 software. The data were expressed as the means \pm standard deviation. For continuous variables, Student's t-test and ANOVA were used to determine the significance of differences among groups. MannWhitney U-test was used for comparing tumor volumes. For categorical variables, Pearson's chi-square test, continuity correction, or Fisher's exact or likelihood ratio were used. A p-value less than 0.05 was considered to be statistically significant.

\section{Results}

Apatinib inhibited ESCC cell proliferation, migration and invasion

To explore the effect of apatinib on the proliferation of ESCC cells, we treated the cells with 7 different apatinib concentrations and evaluated the cells at 3 different time points. The results showed that apatinib significantly inhibited the proliferation of ESCC cells (Fig. 1a, b). IC50 values were calculated, and the results were presented in Fig. 1c. Next, plate clone formation experiments were conducted to verify our conclusions. The results showed that apatinib significantly inhibited colony formation (Fig. 1d-f). In addition, the effect of apatinib on the invasion and

Table 1 Animal experimental groups

\begin{tabular}{lll}
\hline Name of groups & Numbers of animals & Drug intervention \\
\hline Control group & 4 & Use PBS only \\
Apatinib group & 4 & $60 \mathrm{mg} / \mathrm{kg}$, daily oral gavage \\
CQ group & 4 & $60 \mathrm{mg} / \mathrm{kg}$, daily oral gavage \\
Apatinib + CQ group & 4 & $60 \mathrm{mg} / \mathrm{kg}$, daily oral gavage of both drugs \\
Paclitaxel group & 4 & $15 \mathrm{mg} / \mathrm{kg}$, twice a week, intraperitoneal injection \\
Apatinib + CQ + Paclitaxel group & 4 & Apatinib and CQ: $60 \mathrm{mg} / \mathrm{kg}$, daily oral gavage; \\
& & Paclitaxel: $15 \mathrm{mg} / \mathrm{kg}$, twice a week, intraperitoneal \\
\end{tabular}




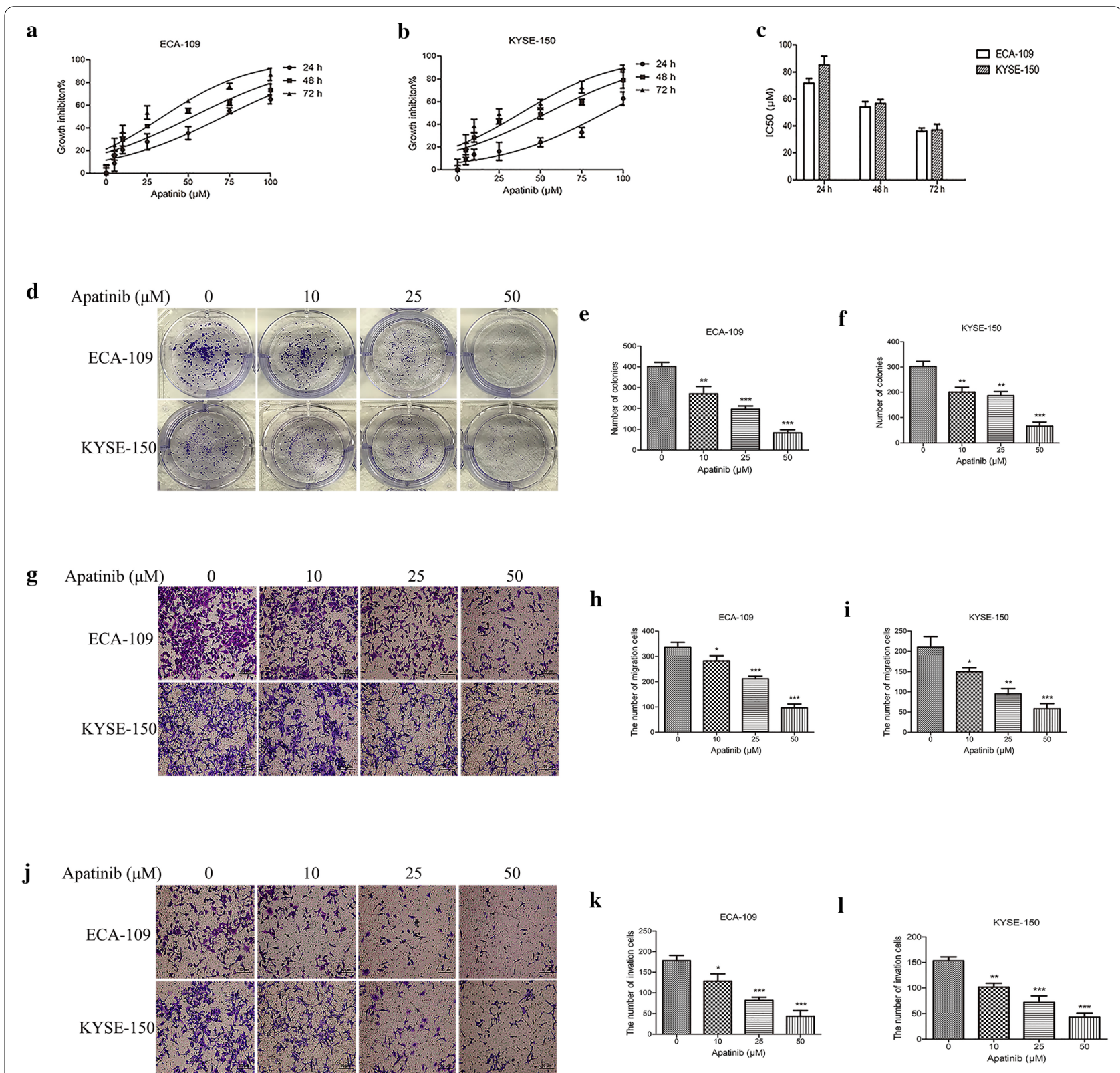

Fig. 1 Apatinib inhibited the proliferation, migration and invasion of ESCC cells. $\mathbf{a}$, b Cell viability was evaluated by CCK8 assay. c IC50 values were calculated. $\mathbf{d}$-f Representative images and quantification of clone formation experiments were shown. $\mathbf{g}-\mathbf{i}$ Apatinib inhibited the migration of ESCC cells. $\mathbf{j}-\mathbf{I}$ Apatinib inhibited the invasion of ESCC cells. ${ }^{*} p<0.05,{ }^{* *} p<0.01,{ }^{* * *} p<0.001$ compared with control group

migration of ESCC cells was explored. The results showed that apatinib significantly inhibited the invasion and migration ability of tumor cells in a dosedependent manner (Fig. 1g-l). In summary, apatinib inhibited the proliferation, invasion and migration of ESCC cells.

\section{Apatinib arrested the cell cycle and induced apoptosis}

Then, we tried to elucidate the mechanism by which apatinib inhibited the proliferation of ESCC cells. We firstly used flow cytometry to study the effect of apatinib on the cell cycle. The results indicated an evident decrease in cell numbers in the $\mathrm{S}$ phase, while 
a

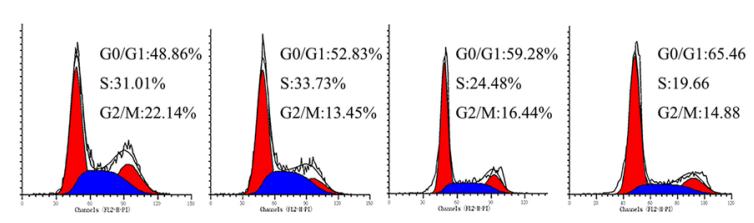

KYSE-150

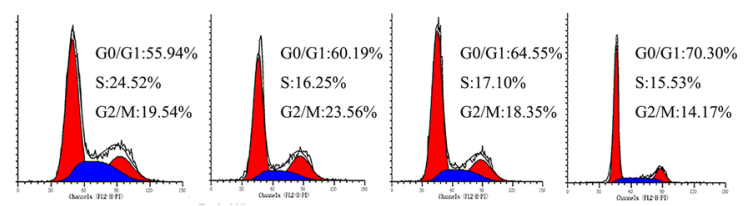

d

Apainib $(\mu \mathrm{M}) \quad 0$

ECA-109
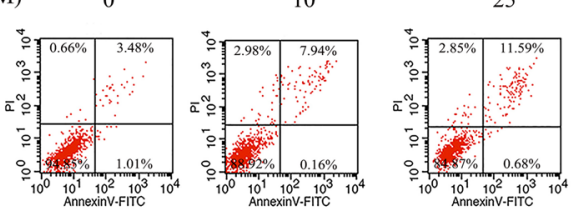

KYSE-150
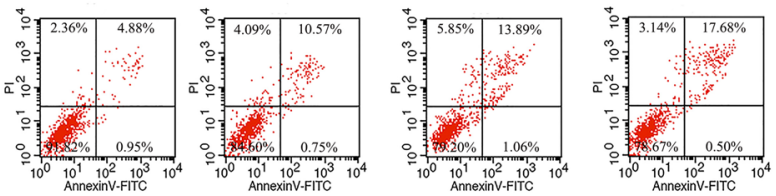

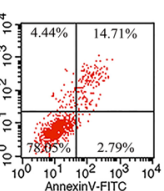

b
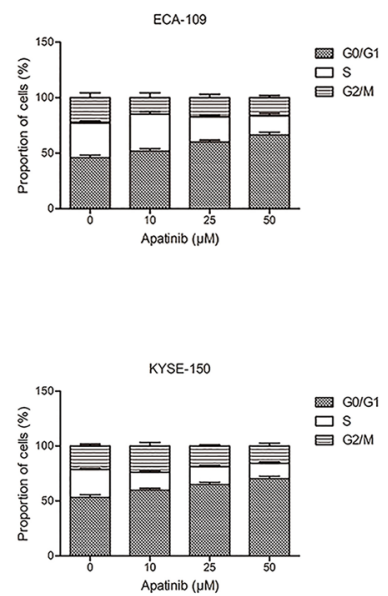

e

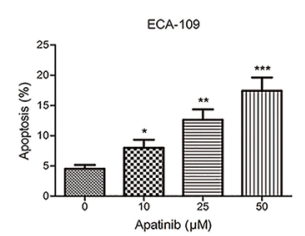

f

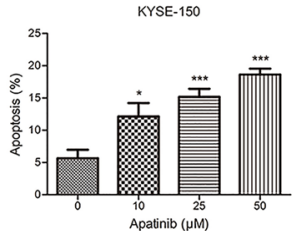

g

ECA-109

Apatinib $(\mu \mathrm{M})$

Bax

$\mathrm{Bcl} 2$

Caspase 3

Cleaved-

Caspase3

PARP

Cleaved-

PARP

GAPDH
KYSE-150

$\begin{array}{llll}0 & 10 & 25 & 50\end{array}$
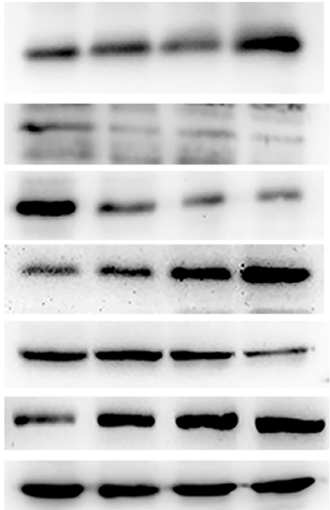

h

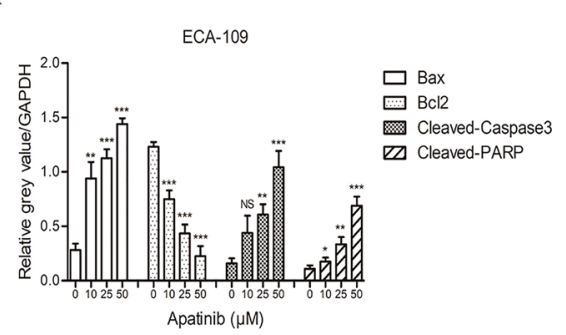

i

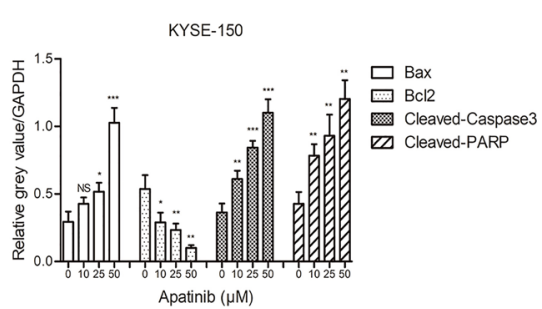

Fig. 2 Apatinib induced cell cycle arrest and the apoptosis of ESCC cells. a-c Cell cycle phase distribution was measured by flow cytometry. $\mathbf{d}-\mathbf{f}$ Annexin V-FITC and propidium iodide (PI) staining were used to measure apoptotic ESCC cells treated with apatinib for $24 \mathrm{~h}$. g-i Western blot analysis was performed to measure the expression levels of apoptosis markers after cells were incubated with apatinib for $24 \mathrm{~h} .{ }^{*} p<0.05$, ** $p<0.01,{ }^{* * *} p<0.001$ compared with control group 
the number of cells in the G0/G1 phase markedly increased after treatment with apatinib (ECA-109 cells $59.89 \pm 1.61$ (apatinib $25 \mu \mathrm{M}$ ) vs $45.83 \pm 1.95$ (control), $\mathrm{p}<0.01,66.39 \pm 2.03$ (apatinib $50 \mu \mathrm{M}$ ) vs $45.83 \pm 1.95$ (control), p $<0.001$; KYSE-150 64.98 \pm 1.58 (apatinib $25 \mu \mathrm{M}$ ) vs $53.21 \pm 1.98$ (control), $\mathrm{p}<0.01,70.20 \pm 1.84$ (apatinib $50 \mu \mathrm{M}$ ) vs $53.21 \pm 1.98$ (control), p $<0.001$ ) (Fig. $2 \mathrm{a}-\mathrm{c}$ ). These results showed that apatinib inhibited the G1-S transition of ESCC cells, thereby inhibiting cell proliferation. We secondly explored the effects of apatinib on the apoptosis of ESCC cells. Flow cytometry and western blotting were used to evaluate the apoptosis rate. The results of flow cytometry revealed that apatinib significantly increased the proportion of apoptotic cells compared with the control group (Fig. 2d-f). The western blot results showed that the expression levels of Bax, Cleaved-Caspase3, and Cleaved-PARP were significantly increased, while the expression levels of $\mathrm{Bcl} 2$, Caspase3, and PARP levels were decreased after treatment with apatinib (Fig. 2g-i).

\section{Apatinib induced ER stress and autophagosome formation} Apoptosis has been reported to be closely related to ER stress and autophagy [23]. Therefore, we focused on the effect of apatinib on ER stress and autophagy in ESCC. Figure 3a showed that compared with the control group, the rough ER in the apatinib group was expanded, the surface ribosomes were partially shed, the Golgi apparatus showed severe hypertrophy, and more autophagic lysosomes were formed in the cytoplasm, indicating that both ER stress and autophagy had been activated. Then, we verified the occurrence of the two phenomena. For assessing ER stress, western blot analysis was performed to detect UPR-targeting markers including Chop, GRP78, IRE-1 $\alpha$ and JNK. The results showed that the expression of these markers was significantly up-regulated after treatment with apatinib (Fig. 3b-d). Maintenance of calcium homeostasis is one of the crucial functions of the endoplasmic reticulum. When ER stress occurs, the calcium ion balance is obviously disordered. Therefore, we further evaluated the level of calcium in the cytoplasm. Fluo3AM was used to label calcium ions, which were subsequently detected by flow cytometry, and the results showed that the level of calcium ions significantly increased after cell treatment with apatinib (Fig. 3e-g). For assessing autophagy, we first performed a western blot analysis to detect the expression levels of classic markers, such as LC3, Beclin 1 , and P62, and the results were shown in Fig. $3 \mathrm{~h}-\mathrm{j}$. The addition of apatinib significantly upregulated the expression levels of LC3 II and Beclin1 and inhibited P62 expression, compared with the control group. However, since autophagy was a dynamic process, we were not sure whether apatinib activated early autophagy or suppressed late autophagy; therefore, BafA1 was used for further exploration. Figure $3 \mathrm{k}-\mathrm{m}$ showed that the upregulation effect of apatinib on LC3 expression was more obvious when apatinib was combined with BafA1. Then, the RFP-GFP-LC3 fusion gene lentivirus was used to transfect ESCC cells. In the analysis, yellow dots represented autophagosomes and red dots represented autolysosomes. The results showed that the number of yellow and red dots increased significantly after treatment with apatinib, suggesting that apatinib significantly increased autophagic flux (Fig. 3n-r). These results indicated that ER stress and autophagy were activated by apatinib in the ESCC cells.

Next, we further explored the specific mechanism by which apatinib affected autophagy and apoptosis in ESCC cells. It has been reported that ER stress was related to both autophagy and apoptosis [23], and

\footnotetext{
(See figure on next page.)

Fig. 3 Apatinib induced ER stress and autophagy in ESCC cells. a Electron microscopy was used to view changes in the rough endoplasmic reticulum, formation of vacuolization and the number of autophagosomes and/or autolysosomes. $N$ nucleus, $M$ mitochondria, RER rough endoplasmic reticulum, ASS autolysosome, GO Golgi apparatus, LD lipid droplet. b-d Western blot analysis was performed to measure the expression levels of ER stress markers after cells were incubated with apatinib for $24 \mathrm{~h}$. $\mathbf{e}-\mathbf{g}$ The level of cytosolic calcium was evaluated by flow cytometry. $\mathbf{h}-\mathbf{j}$ The expression levels of autophagy markers in cells incubated with apatinib for $24 \mathrm{~h}$ were measured by western blotting. $\mathbf{k}-\mathbf{m}$ The expression levels of LC-3, P62, and GAPDH in cells treated with $25 \mu \mathrm{M}$ apatinib for $24 \mathrm{~h}$ or $50 \mathrm{nM}$ BafA 1 for $12 \mathrm{~h}$ were measured by western blotting. $\mathbf{n}-\mathbf{r}$ Representative images and quantification of autophagosomes shown as yellow dots and autolysosomes shown as red dots were shown in cells transfected with RFP-GFP-LC3 lentivirus and incubated with $25 \mu \mathrm{M}$ apatinib for $24 \mathrm{~h} .{ }^{*} p<0.05,{ }^{* *} p<0.01,{ }^{* * *} p<0.001$ compared with control group
} 

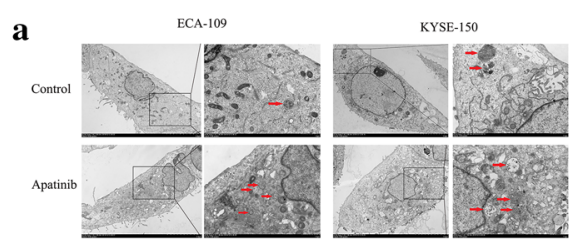

b
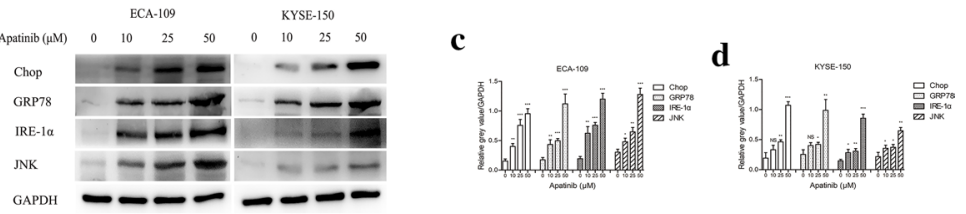

e

Apatinib $(\mu \mathrm{M})$
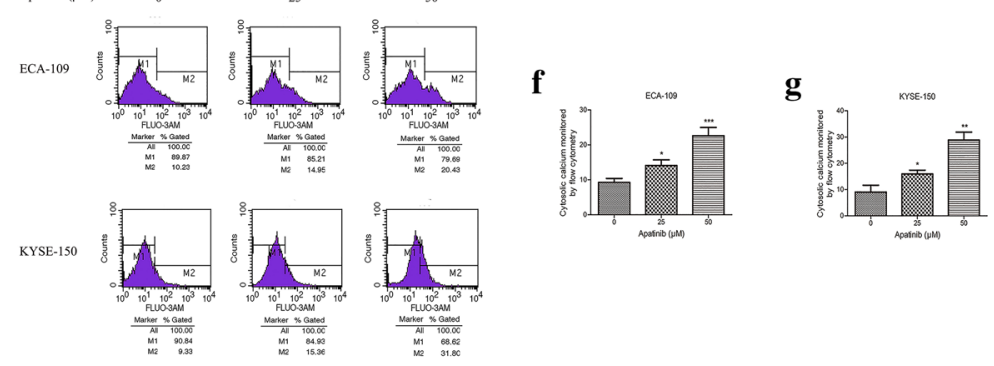

h
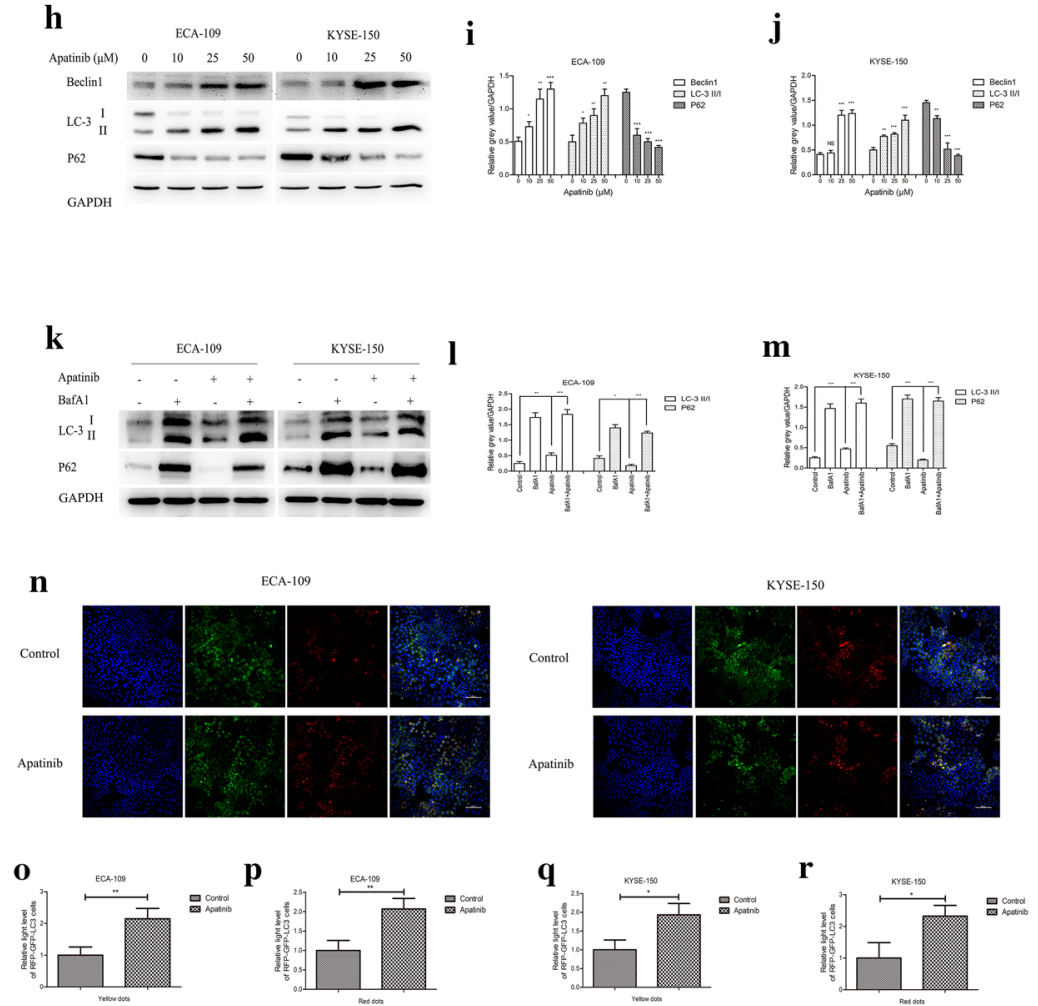

Fig. 3 (See legend on previous page.) 
the IRE- $1 \alpha$ pathway was one of the three main pathways involved in ER stress. Many studies have shown that the AKT-mTOR pathway was closely related to autophagy and apoptosis [27, 28], but it remained unclear whether IRE- $1 \alpha$ mediated the autophagy and/ or apoptosis induced by apatinib through the AKTmTOR pathway. IRE-1 $\alpha$ siRNA was used to transfect ESCC cell lines. Figure $4 \mathrm{a}-\mathrm{g}$ showed that knocking down IRE-1 $\alpha$ inhibited the expression levels of ER stress- and autophagy-related proteins. In addition, knocking down IRE- $1 \alpha$ also had a significant impact on apoptosis-related proteins (Fig. $4 \mathrm{a}-\mathrm{g}$ ). More interestingly, we found that after silencing IRE- $1 \alpha$, the activity levels of pAKT and pmTOR were also reduced, which suggested that IRE1- $\alpha$ might affect the regulation of autophagy and apoptosis by apatinib through the AKT-mTOR pathway (Fig. $4 \mathrm{a}-\mathrm{g}$ ). Then, the AKT activator SC79 was used alone or in combination with apatinib. The western blot results showed that compared with apatinib, the combination of the two drugs significantly inhibited the expression levels of Bax and LC3 II, but increased the expression levels of $\mathrm{Bcl} 2$, p62, pAKT and pmTOR which indicated that SC79 inhibited the activation of apatinib on autophagy and apoptosis (Fig. 4h-l). Flow cytometry was carried out to detect apoptotic cells. Figure $4 \mathrm{~m}-\mathrm{o}$ showed that compared with apatinib treatment alone, the combination of apatinib and SC79 significantly inhibited apoptosis. Rapamycin, an inhibitor of mTOR, was used to explore the influence of the mTOR pathway on the effect of apatinib. The western blot results showed that compared with apatinib treatment alone, the combination of apatinib and rapamycin inhibited the expression of $\mathrm{Bcl} 2$ but increased the expression levels of Bax and LC3 II (Fig. 4p-r). The results of the apoptosis detection assay by flow cytometry showed that compared with apatinib treatment alone, the combination of apatinib and rapamycin further increased the tumor cell apoptosis rate (Fig. $4 \mathrm{~s}-\mathrm{u})$. These results indicated that the IRE- $1 \alpha-\mathrm{AKT}-\mathrm{mTOR}$ pathway was involved in the regulation of autophagy and apoptosis by apatinib.

\section{Inhibiting autophagy increased apatinib-induced apoptosis}

The aforementioned results indicated that apatinib regulated autophagy and apoptosis through the IRE-1 $\alpha-A K T-$ mTOR pathway, but it remained unclear whether autophagy played a role in this process. First, Beclin1 siRNA was used to knock down Beclin1, and then, the effect of apatinib on the apoptosis of ESCC cells was determined. The western blot results showed that the expression of Bax was significantly increased after Beclin1 was knocked down, while the expression of $\mathrm{Bcl} 2$ was significantly decreased (Fig. $5 \mathrm{a}-\mathrm{c}$ ). CQ, an antimalarial lysosomal inhibitor, has been identified as an inhibitor of autophagy [29], and it has been found to prevent autophagy by blocking autophagosomal-lysosomal fusion [30]. The western blot results revealed that administration of $C Q$ as a pretreatment led to significant accumulation of lipidated LC3 and P62, indicating that autophagy had been inhibited. Compared with apatinib treatment alone, the combination of CQ and apatinib significantly increased the expression of Bax and inhibited the expression levels of Bcl2, pAKT and pmTOR, which suggested that the inhibition of autophagy enhanced the effect of apatinib on tumor cell apoptosis (Fig. $5 \mathrm{~d}-\mathrm{h}$ ). We used the RFP-GFP-LC3 lentivirus to transfect ESCC cells. The results of confocal microscopy indicated that compared with apatinib treatment alone, the combination of CQ and apatinib significantly increased the number of yellow spots, while the number of red spots decreased, indicating that the formation of autophagolysosomes was inhibited by CQ (Fig. 5i-n). Finally, flow cytometry was used to detect apoptotic cells.

(See figure on next page.)

Fig. 4 Apatinib affected autophagy and apoptosis via the IRE-1 a-AKT-mTOR pathway. a-g ECA-109 and KYSE-150 cells were treated with $25 \mu \mathrm{M}$ apatinib or DMSO for $24 \mathrm{~h}$ after transfection with control siRNA or IRE-1 a siRNA. The expression levels of related genes were measured by western blotting. $\mathbf{h}-\mathbf{o}$ Cells were treated with apatinib $(25 \mu \mathrm{M})$ with or without SC79 $(4 \mu \mathrm{g} / \mathrm{ml})$. The expression levels of related genes were measured by western blotting ( $\mathbf{h}-\mathbf{l})$. The number of apoptotic cells was determined by Annexin V-FITC and propidium iodide (PI) staining ( $\mathbf{m}-\mathbf{0})$. $\mathbf{p}-\mathbf{u}$ Cells were treated with apatinib $(25 \mu \mathrm{M})$ with or without rapamycin $(1 \mathrm{nM})$. The expression levels of related genes were measured by western blotting (p-r). The number of apoptotic cells was assessed by Annexin V-FITC and propidium iodide (PI) staining (s-u). ${ }^{*} p<0.05,{ }^{* *} p<0.01,{ }^{* * *} p<0.001$ compared with control group. ${ }^{\#} p<0.05,{ }^{\# \#} p<0.01, \# \#<0.001$ 


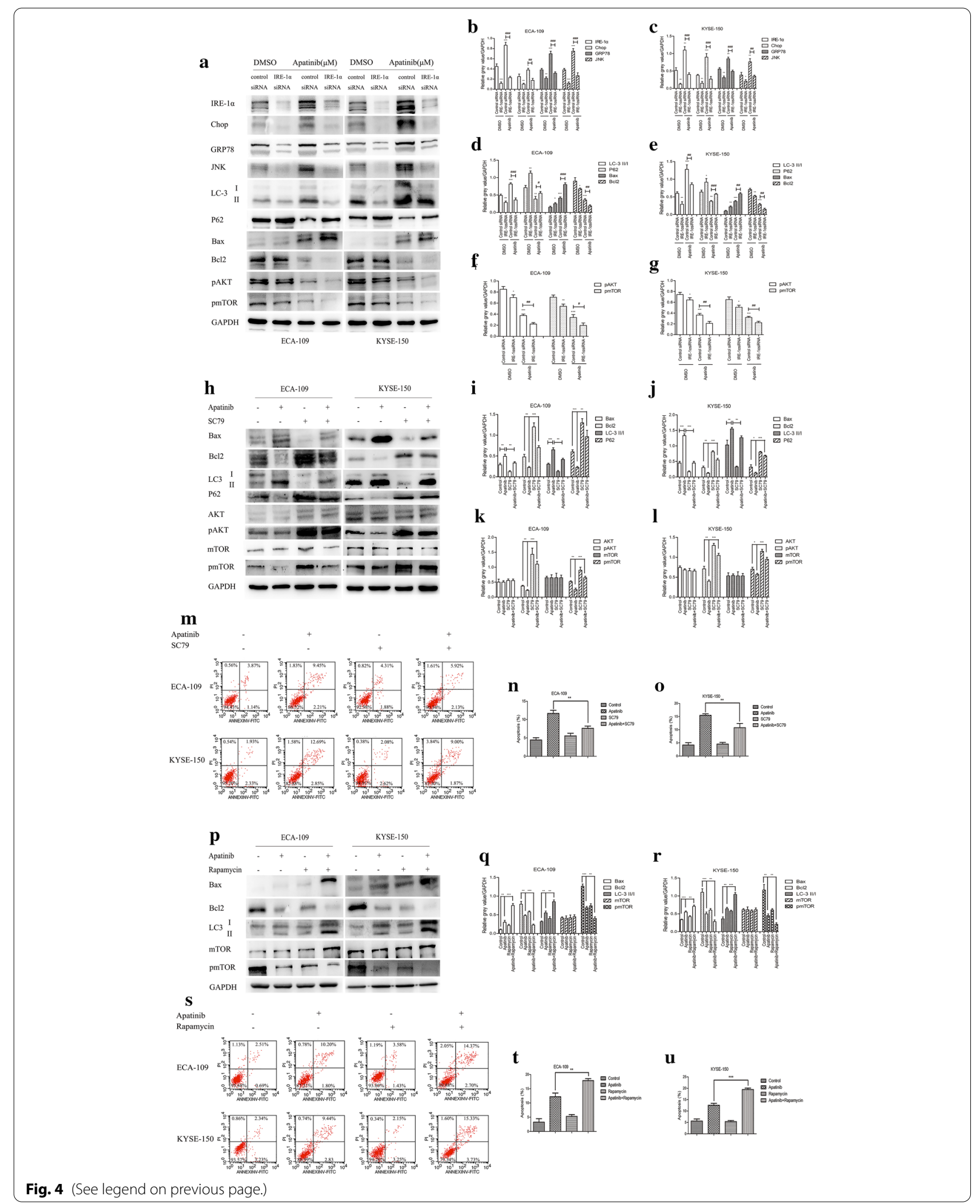


Figure $50-\mathrm{q}$ showed that the administration of $\mathrm{CQ}$ as a pretreatment significantly enhanced the induction of apoptosis by apatinib. In summary, we demonstrated that inhibition of autophagy enhanced the inducing effect of apatinib on tumor cell apoptosis.

\section{The combination of apatinib and CQ sensitized cells to paclitaxel to inhibit their proliferation and induce apoptosis}

We further explored whether the combination of apatinib and CQ could sensitize cells to paclitaxel. Different concentrations of paclitaxel were used to treat ESCC cells with or without apatinib and CQ for 24,48 , and $72 \mathrm{~h}$. The results indicated that apatinib and CQ significantly enhanced the inhibitory effect of paclitaxel on the proliferation of tumor cells and reduced the paclitaxel IC50 values (Fig. 6a-h). Plate clone formation experiments were used to further verify our conclusions. Compared with the effect of paclitaxel treatment alone, the combination of paclitaxel with apatinib and CQ significantly inhibited the formation of ESCC clones (Fig. 6i-k). Flow cytometry was used to evaluate the apoptosis rate of tumor cells. Figure 6l-n showed that apatinib and CQ significantly enhanced the rate of tumor cell apoptosis induced by paclitaxel. The western blot results revealed that when apatinib and CQ were combined with paclitaxel, the expression levels of Bax and Cleaved-Caspase 3 were significantly increased, while the levels of $\mathrm{Bcl} 2, \mathrm{pAKT}$ and pmTOR were significantly reduced (Fig. 6o-q). These results suggested that apatinib and CQ could significantly enhance the inhibitory effect of paclitaxel on tumor cell proliferation and accelerate apoptosis.

\section{The IRE-1a-AKT-mTOR signaling pathway participated} in the sensitization of cells to paclitaxel through the effects of apatinib and CQ

The results shown in Fig. 6o-q indicated that the combination of paclitaxel with apatinib and CQ further inhibited the expression levels of $\mathrm{pAKT}$ and
pmTOR; however, it remained unknown whether the sensitization effect of apatinib and CQ on cell sensitization to paclitaxel mediated through the IRE-1 $\alpha$-AKT-mTOR pathway. IRE-1 $\alpha$ siRNA was first transfected into ESCC cell lines, which were then treated with different drugs for $24 \mathrm{~h}$ after transfection. The western blot results showed that after knocking down IRE-1 $\alpha$, compared with the combination of paclitaxel with apatinib and CQ group, the expression level of Bax was further increased, and the expression levels of $\mathrm{Bcl} 2$, pAKT and pmTOR were further decreased in the IRE-1 $\alpha$-knockdown group (Fig. 7ae). This result revealed that IRE- $1 \alpha$ was involved in the sensitization of paclitaxel by apatinib and CQ, and was the upstream molecule of the AKT-mTOR pathway. SC79, an AKT activator, was used in combination with apatinib and CQ and paclitaxel or not. The western blot results showed that compared with the combination of paclitaxel with apatinib and CQ group, the addition of $\mathrm{SC79}$ to this combination reduced the expression levels of Bax and Cleaved-Caspase 3 and increased the expression levels of $\mathrm{Bcl} 2, \mathrm{pAKT}$ and pmTOR (Fig. $7 \mathrm{f}-\mathrm{j}$ ). The results of the apoptosis assay with flow cytometry verified that the addition of SC79 would reverse the inducing effect of paclitaxel combined with apatinib and CQ on the apoptosis of ESCC cells (Fig. 7k-m). Figure $7 n-r$ showed the effect of the mTOR inhibitor rapamycin on the apoptosis induced by paclitaxel combined with apatinib and CQ. The western blot results showed that compared with paclitaxel combined with apatinib and CQ group, adding rapamycin to this combination further increased the expression levels of Bax and Cleaved-Caspase3, while the expression levels of $\mathrm{Bcl} 2$ and pmTOR were reduced. These results corroborated the findings showing that the facilitating effect of apatinib and CQ on cell sensitization to paclitaxel was regulated by the IRE-1 $\alpha-$ AKT-mTOR pathway.

(See figure on next page.)

Fig. 5 Inhibiting autophagy increased apatinib-induced apoptosis. a-c Cells were treated with $25 \mu \mathrm{M}$ apatinib or DMSO for $24 \mathrm{~h}$ after transfection with control siRNA or Beclin1 siRNA. The expression levels of related genes were measured by western blotting. $\mathbf{d}-\mathbf{q}$ Cells were pretreated with $10 \mu \mathrm{M}$ CQ for $6 \mathrm{~h}$ and then treated with apatinib $(25 \mu \mathrm{M})$ for $24 \mathrm{~h}$. The expression levels of related genes were detected by western blotting $(\mathbf{d}-\mathbf{h})$. Representative images and quantification of autophagosomes shown as yellow dots and autolysosomes shown as red dots were presented $(\mathbf{i}-\mathbf{n})$. The number of apoptotic cells was assessed by Annexin V-FITC and propidium iodide (PI) staining (o-q). ${ }^{*} p<0.05,{ }^{* *} p<0.01,{ }^{* * *} p<0.001$ compared with control group. ${ }^{\prime} p<0.05,{ }^{\# \#} p<0.01,{ }^{\# \#} p<0.001$ 


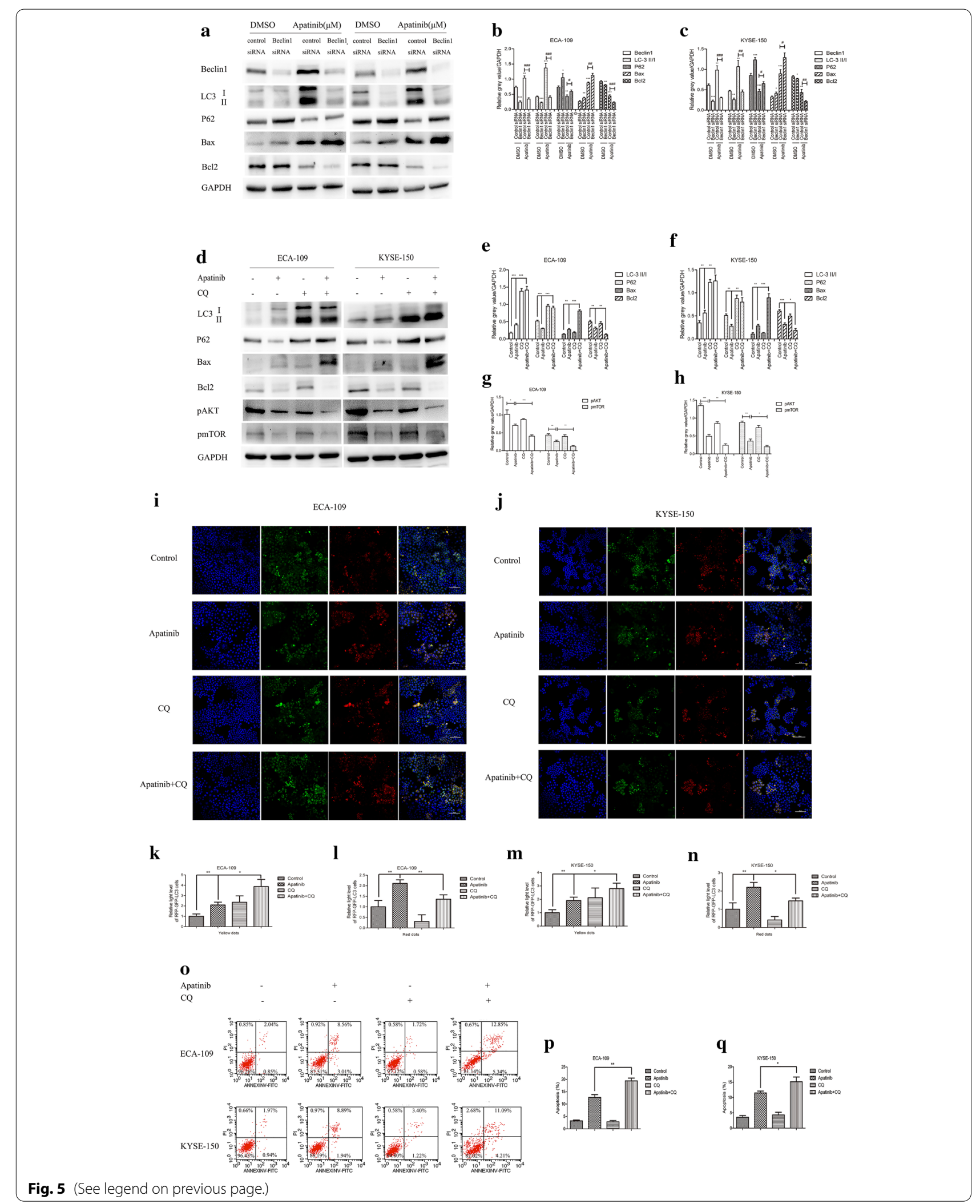




\section{The combination of apatinib and CQ intensified tumor suppression induced by paclitaxel}

Considering the aforementioned results, we verified our conclusions through in vivo experiments. The ECA-109 cell line was used to inoculate nude mice subcutaneously. After two weeks, all the nude mice were assigned to 6 groups: control group, CQ group, apatinib group, apatinib and CQ group, paclitaxel group, paclitaxel combined with apatinib and CQ group. Four weeks after the drugs administration, all the mice were sacrificed, and the tumors were removed to measure the volume and weight. Figure $8 \mathrm{a}-\mathrm{c}$ showed that compared with the effect of apatinib alone, the combination of CQ and apatinib significantly reduced tumor volume and weight $\left(307.00 \pm 42.01 \mathrm{~mm}^{3}\right.$ (apatinib $\left.+\mathrm{CQ}\right)$ vs $693.33 \pm 67.99$ $\mathrm{mm}^{3}$ (apatinib), $\mathrm{p}<0.01 ; 533.33 \pm 108.73 \mathrm{~g}$ (apatinib + CQ) vs. $906.67 \pm 70.40$ g (apatinib), $\mathrm{p}<0.05$ ). Compared with the control group mice, the paclitaxel group mice exhibited reduced tumor volume and weight to a certain extent $\left(417.33 \pm 24.78 \mathrm{~mm}^{3}\right.$ (paclitaxel) vs $1547.33 \pm 47.79 \mathrm{~mm}^{3}$ (control), p $<0.001$; $570.00 \pm 106.14 \mathrm{~g}$ (paclitaxel) vs $1533.33 \pm 164.99 \mathrm{~g}$ (control), $\mathrm{p}<0.01$ ), and when paclitaxel was combined with apatinib and CQ, the reducing effects on tumor volume and weight were most obvious $(86.83 \pm 24.29$ $\mathrm{mm}^{3}$ (paclitaxel + apatinib +CQ) vs $417.33 \pm 24.78$ $\mathrm{mm}^{3}$ (paclitaxel), $\mathrm{p}<0.001 ; 192.33 \pm 27.88 \mathrm{~g}$ (paclitaxel+apatinib $+\mathrm{CQ}$ ) vs $\quad 570.00 \pm 106.14 \quad \mathrm{~g}$ (paclitaxel), $\mathrm{p}<0.01)$. Immunohistochemistry was used to assess the expression of Ki67 and Cleaved-Caspase3 in different groups of tumor tissues, and TUNEL was used to determine the number of apoptotic cells in different groups of tumor tissues. Figure $8 d-g$ showed that, compared with the apatinib group, the CQ and apatinib group showed more significantly inhibited Ki67 and Cleaved-Caspase3 expression and increased apoptosis in tumor tissue. Compared with the paclitaxel single-agent group, the paclitaxel combined with apatinib and CQ group significantly inhibited Ki67 expression and increased Cleaved- Caspase 3 expression, and the apoptosis rate in tumor tissue was also increased significantly. These results corroborated the finding that apatinib and CQ improved the ability of paclitaxel to inhibit cell proliferation and promote apoptosis in ESCC tissues.

\section{Discussion}

Esophageal squamous cell carcinoma is a major lifethreatening malignancy characterized by high incidence and mortality, and early distant metastasis [31, 32]. Surgery plays a pivotal role in the treatment of $\mathrm{T} 1$ or T2 stage tumors, while neoadjuvant/adjuvant therapy is applied for locally advanced ESCC $[33,34]$. Since ESCC is often detected at an advanced stage, chemotherapy and targeted therapy might be effective perioperative treatments [7]. At present, there are many types of drugs involved in targeted therapy of ESCC, but most researches about targeted therapy are still in the clinical or basic research stage and only 3 targeted therapeutic agents including trastuzumab, ramucirumab, and pembrolizumab, have been approved by the FDA for use in esophageal and EGJ cancers. In many studies, apatinib has been reported not only to exert antitumor effects but also to enhance the sensitivity of tumor cells to chemotherapeutic agents. Huang et al. reported that apatinib inhibited the proliferation, migration and invasion of cholangiocarcinoma cells [35]. Meng et al. found that apatinib inhibited cell proliferation and induced autophagy in human papillary thyroid carcinoma [36]. Xu et al. proved that apatinib enhanced the chemosensitivity of gastric cancer cells to paclitaxel and 5-fluorouracil [37]. Moreover, Li et al. found that apatinib notably enhanced the antitumor activity of gefitinib in NSCLC cells and in xenografts carrying T790M mutations [38]. So far, there have been few studies on apatinib application in the treatment of ESCC. Wei et al.

\footnotetext{
(See figure on next page.)

Fig. 6 The combination of apatinib and CQ sensitized ESCC cells to paclitaxel, which inhibited their proliferation and induced apoptosis. a-f ECA-109 and KYSE-150 cells were treated with different concentrations of paclitaxel with or without apatinib $(25 \mu \mathrm{M})$ and CQ $(10 \mu \mathrm{M})$ for 24,48 and $72 \mathrm{~h}$ and cell viability was evaluated by CCK-8 assay. $\mathbf{g}, \mathbf{h}$ IC50 values were calculated. $\mathbf{i}-\mathbf{k}$ Representative images of the colonies formed and colony quantification were shown. Cells were treated with paclitaxel $(5 \mathrm{nM})$ with or without apatinib $(25 \mu \mathrm{M})$ and CQ $(10 \mu \mathrm{M})$. Apoptosis was assessed by Annexin V-FITC and propidium iodide (PI) staining (I-n). Western blotting was used to measure the expression levels of related genes $(\mathbf{o}-\mathbf{q})$. * $p<0.05,{ }^{* *} p<0.01,{ }^{* * *} p<0.001$ compared with control group
} 

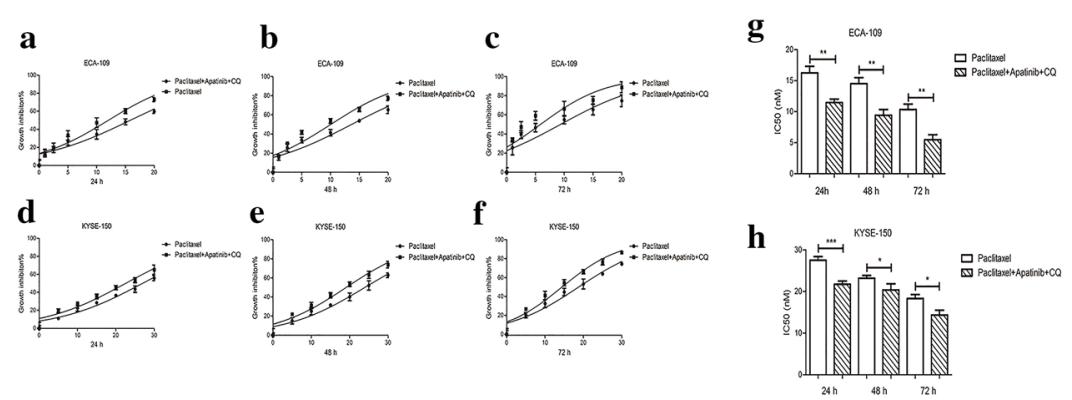

$$
\text { i }
$$

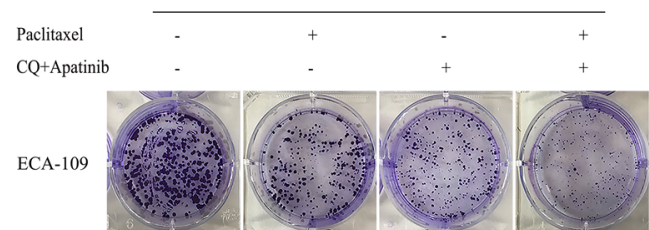

j
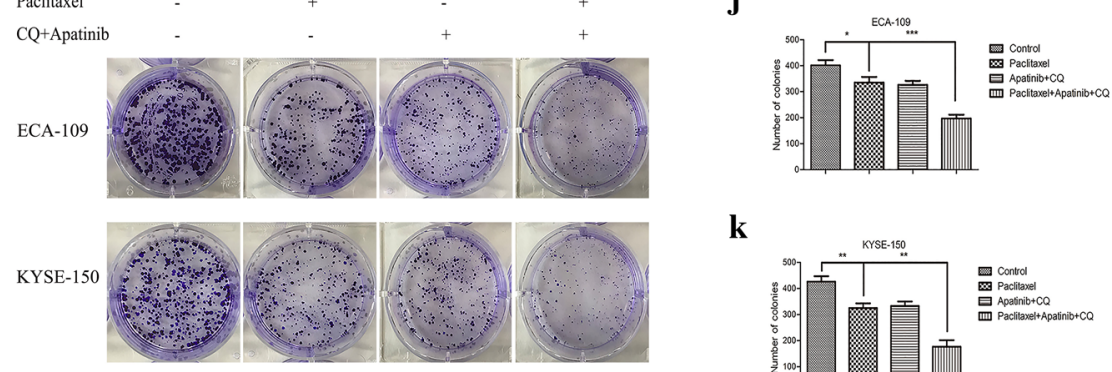

$\mathbf{k}$

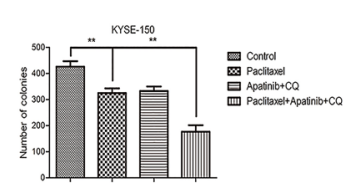

I

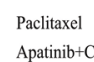

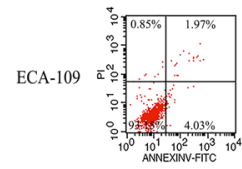
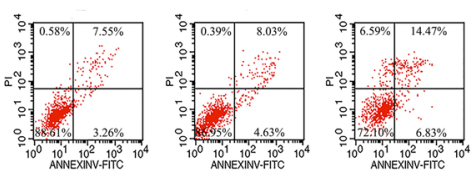

m
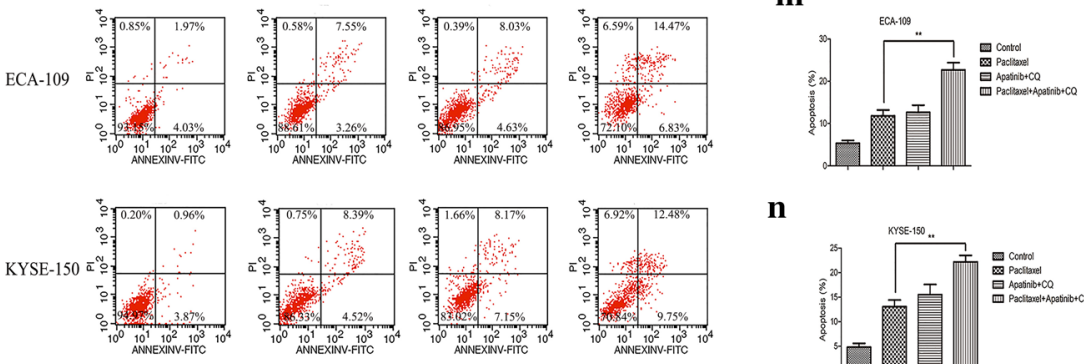

n
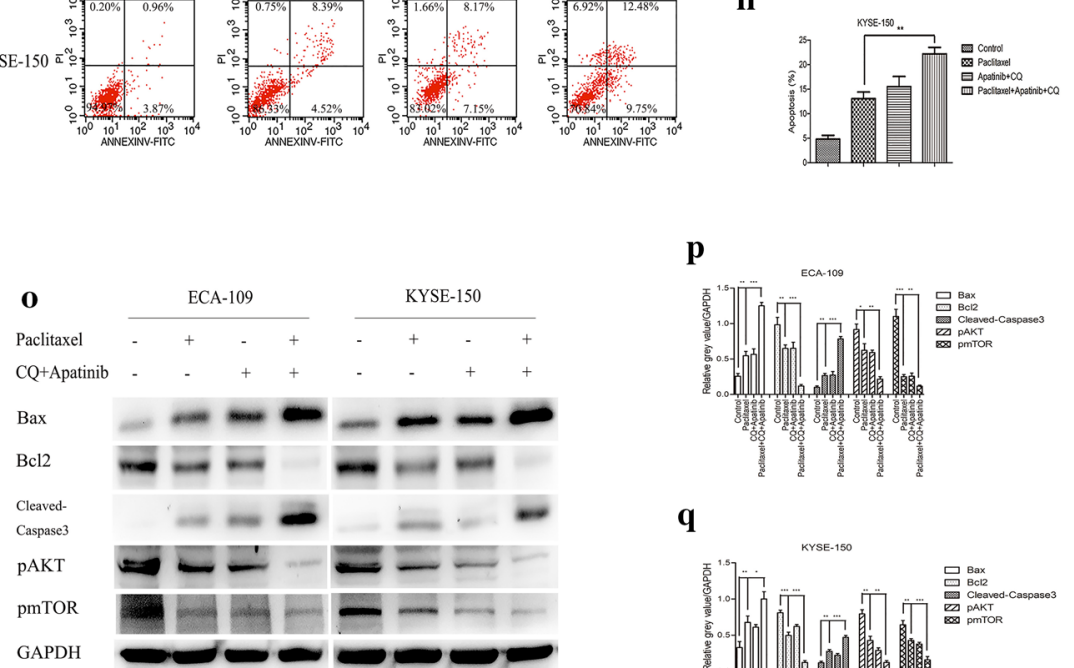

$\mathbf{q}$

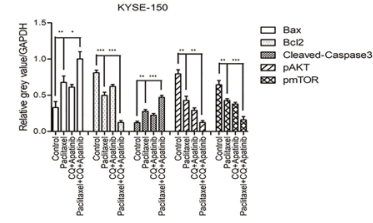

Fig. 6 (See legend on previous page.) 
reported that apatinib suppressed tumor progression and enhanced cisplatin sensitivity in esophageal cancer via the Akt/ $\beta$-catenin pathway [18]. The role of apatinib in ESCC and related mechanisms still need to be further clarified. And whether apatinib had a sensitizing effect on paclitaxel and the underlying mechanism in ESCC remained to be explored. In our study, we first clarified the inhibitory effects of apatinib on the cell proliferation, invasion, and migration of ESCC cell lines. Then, we found that apatinib induced ER stress, autophagy and apoptosis. Inhibiting autophagy could enhance apatinib-induced apoptosis. Finally, we proved that the combined application of apatinib and CQ sensitized cells to paclitaxel, which induced apoptosis through the IRE- $1 \alpha-\mathrm{AKT}-\mathrm{mTOR}$ signaling pathway.

It has been reported that there was a close relationship among ER stress, autophagy and apoptosis [39-41]. Generally, autophagy is activated under ER stress. Autophagy induced by ER stress plays both survival- and death-promoting roles. Most relevant studies have demonstrated that autophagy was activated to inhibit apoptosis after ER stress [42, 43]. We aimed to clarify the relationship among ER stress, autophagy and apoptosis, because it was essential for the treatment of ESCC. In our study, we found that apatinib induced ER stress, autophagy and apoptosis of ESCC cells. Knockdown of IRE-1 $\alpha$, which was the key gene in one of three major ER stress signaling pathways, inhibited apatinib-induced autophagy and enhanced apatinib-induced apoptosis. This result indicated that ER stress was the upstream mechanism of apatinib-induced autophagy and apoptosis. Moreover, we found that knocking down IRE- $1 \alpha$ enhanced the inhibitory effects of apatinib on pAKT and pmTOR expression levels. The AKT-mTOR signaling pathway has been reported to be closely related to tumor survival [27, 28]. Thus, SC79 (an AKT activator) and Rapamycin (an mTOR inhibitor) were used to clarify the role of the AKT-mTOR signaling pathway in apatinib-induced autophagy and apoptosis. The results showed that inhibiting the AKT-mTOR pathway increased the expression levels of autophagy- and apoptosis-related proteins and was the downstream mechanism of IRE-1 $\alpha$. Beclin1-siRNA and CQ, which were autophagy inhibitors, were used to clarify the relationship between autophagy and apoptosis. The results showed that inhibiting autophagy by CQ or Beclin1-siRNA promoted the apoptosis of ESCC cells induced by apatinib. Through this part of the study, we confirmed that apatinib could activate ER stress, autophagy, and apoptosis. And the activating effect of apatinib on autophagy and apoptosis was dependent on endoplasmic reticulum stress. Furthermore, inhibiting autophagy increased apatinib-induced apoptosis.

Monotherapy with apatinib appeared to be effective only for selected tumors that were highly dependent on VEGF. Relevant studies have proved that combination of anti-angiogenic targeted therapy and conventional chemotherapy might lead to improved response rates in several solid tumors rates, including ovarian cancer, breast cancer and colon cancer [37]. Considering the synergistic effect of apatinib and CQ, we further explored whether the two drugs sensitized cells to paclitaxel. In our study, we demonstrated that the combination of apatinib and CQ enhanced the inhibition of paclitaxel on the proliferation of ESCC cells and promoted apoptosis. The aforementioned results indicated that apatinib regulated autophagy and apoptosis through the IRE- $1 \alpha-A K T-m T O R$ pathway. We wanted to further elucidate the mechanism by which apatinib and CQ sensitized cells to paclitaxel. IRE- $1 \alpha$ siRNA was used to knock down IRE- $1 \alpha$. SC79 was used to activate expression levels of pAKT

\footnotetext{
(See figure on next page.)

Fig. 7 The combination of apatinib and CQ sensitized cells to paclitaxel, which induced their apoptosis via the IRE-1a-AKT-mTOR pathway. a-e ECA-109 and KYSE-150 cells were treated with $5 \mathrm{nM}$ paclitaxel with or without apatinib and CQ for $24 \mathrm{~h}$ after transfection with control siRNA or IRE-1 a siRNA. The expression levels of related genes were measured by western blotting. $\mathbf{f}-\mathbf{m}$ Cells were treated with paclitaxel or apatinib and CQ with or without SC79 $(4 \mathrm{\mu g} / \mathrm{ml})$. The expression levels of related genes were measured by western blotting $(\mathbf{f}-\mathbf{j})$. The number of apoptotic cells was assessed by Annexin V-FITC and propidium iodide (PI) staining $(\mathbf{k}-\mathbf{m})$. $\mathbf{n}-\mathbf{r}$ Cells were treated with paclitaxel or apatinib and CQ with or without rapamycin $(1 \mathrm{nM})$. The expression levels of related genes were measured by western blotting. ${ }^{*} p<0.05,{ }^{* *} p<0.01,{ }^{* * *} p<0.001$ compared with control group. (P, paclitaxel; A, apatinib; C, CQ; R, rapamycin)
} 
$\mathbf{a}$

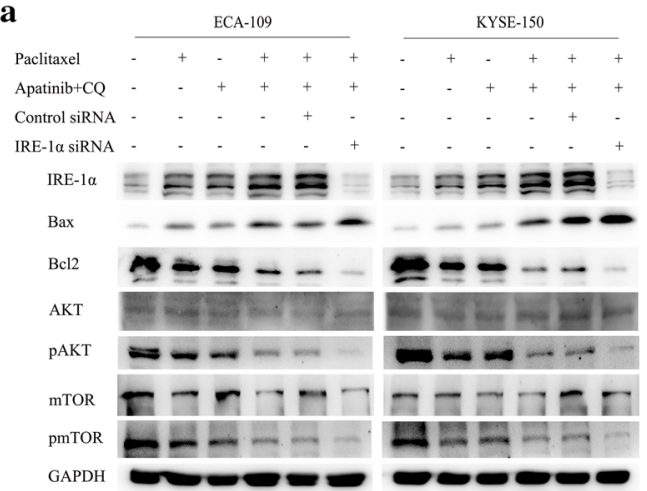

f

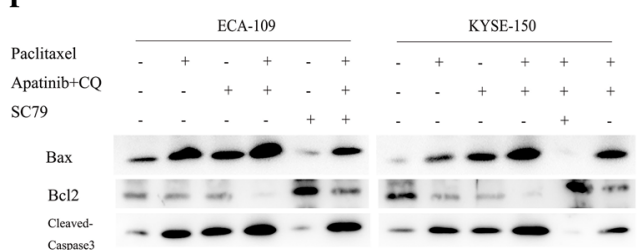

Cleaved. $_{\text {Caspase } 3}-\infty-\infty-\infty-\infty-\infty$

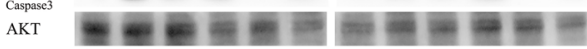

рАКт $-\cdots-\infty-\cdots-\cdots$

mTOR

pmTOR

GAPDH

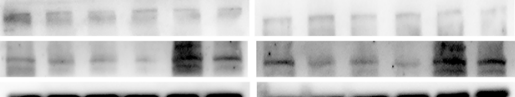

$\mathbf{k}$

Paclitixel
Apatinib+Ce

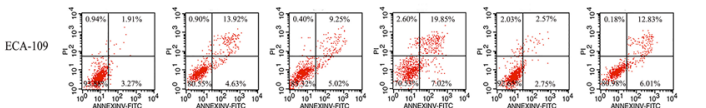

KYSE-150 b

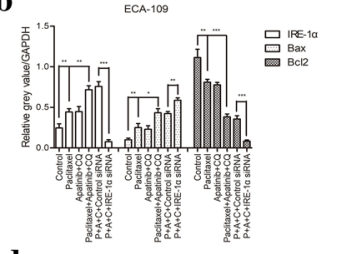

d

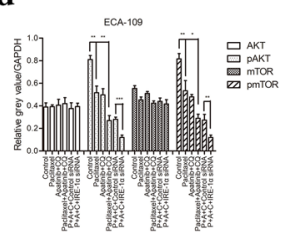

g
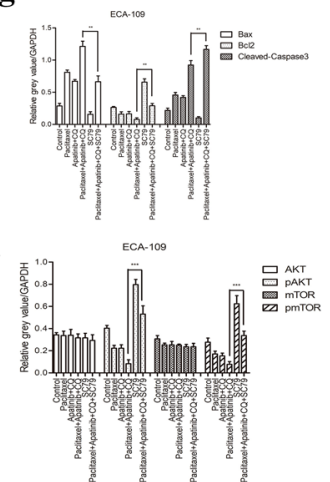

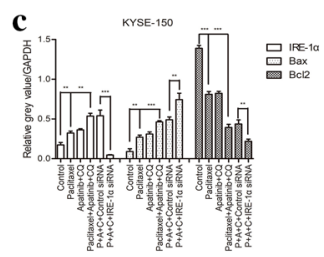

e

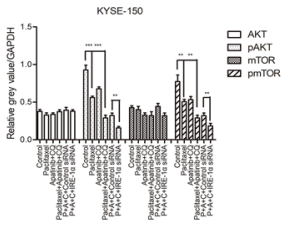

h

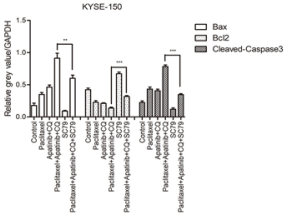

j

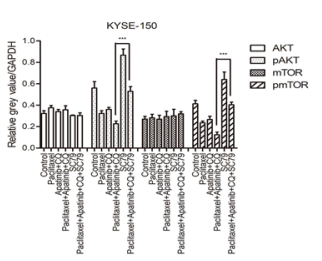

1

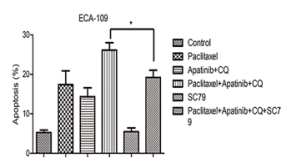

m

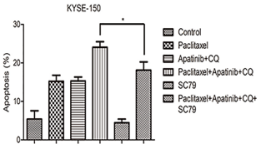

n

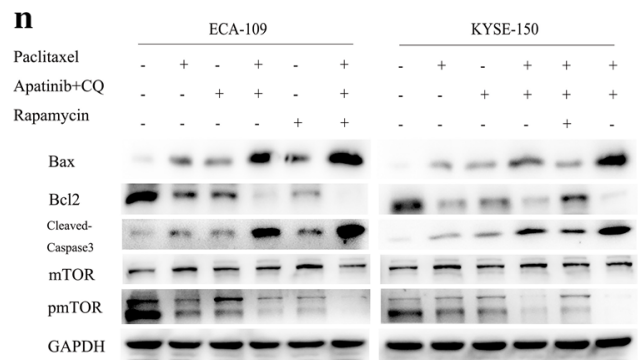

$\mathbf{0}$

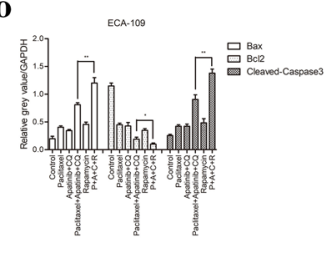

$\mathbf{q}$

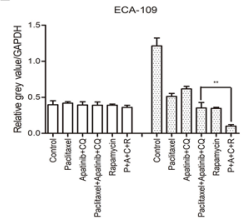

吕 ${ }_{\text {mrToR }}$ $\mathbf{p}$

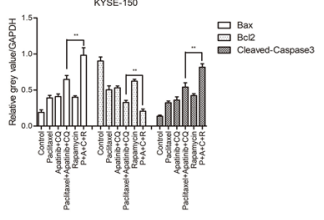

$\mathbf{r}$

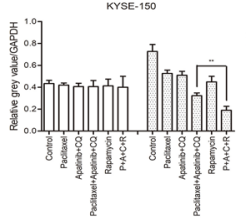

Fig. 7 (See legend on previous page.) 


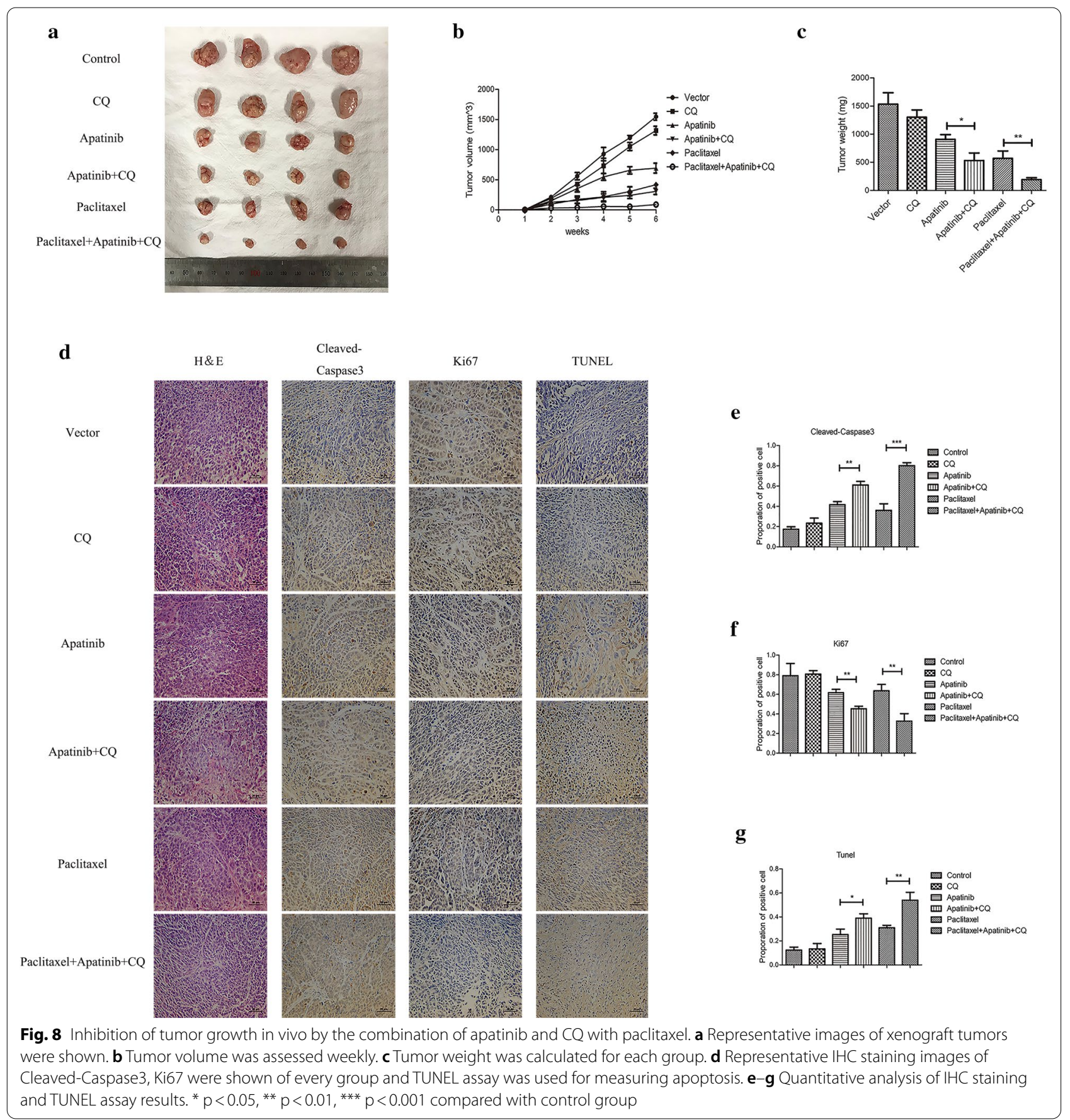

and rapamycin was used to inhibit expression levels of pmTOR. The results showed that the combination of apatinib and CQ sensitized ESCC cells to paclitaxel to induce apoptosis through the IRE-1 $\alpha-\mathrm{AKT}-\mathrm{mTOR}$ signaling pathway both in vivo and in vitro.
In this study, we discovered that apatinib induced ER stress, autophagy and apoptosis in ESCC. Inhibiting autophagy by CQ enhanced apatinib-induced apoptosis. The combination of apatinib and CQ sensitized ESCC cells to paclitaxel to induce apoptosis 
through the IRE-1 $\alpha-\mathrm{AKT}-\mathrm{mTOR}$ signaling pathway. Our study has potential clinical application value and may provide new ideas for the comprehensive diagnosis and treatment of esophageal cancer.

\section{Supplementary Information}

The online version contains supplementary material available at https://doi. org/10.1186/s13578-021-00640-2.

Additional file 1: Figure S1. Construction of IRE-1 $\mathrm{a}$ and Beclin 1 gene knock-down cell models. (a-c) ECA-109 and KYSE-150 cells were transfected with NC-siRNA or IRE-1a-siRNAs for $24 \mathrm{~h}$. The expression level of IRE-1 a was detected by Western blot. (d-f) ECA-109 and KYSE-150 cells were transfected with NC-siRNA or Beclin 1-siRNAs for $24 \mathrm{~h}$. The expression level of Beclin 1 was detected by Western blot.

\section{Acknowledgements}

Not applicable.

\section{Authors' contributions}

QY and XMM designed the study. YMW and XX carried out the experiments and wrote the paper. JT and ZYS contributed to data curation and supervision. YJF and XJZ contributed to the writing-review and revisions. All authors read and approved the final manuscript.

\section{Funding}

This work was supported by the National Natural Science Foundation of China (8207100395) to Xiao-Jing Zhao.

\section{Availability of data and materials}

All data used and/or analyzed in the current study are available from the corresponding author on reasonable request.

\section{Declarations}

\section{Ethics approval and consent to participate}

All procedures were approved by the Animal Care and Use Committee of Renji Hospital affiliated to Shanghai Jiao Tong University School of Medicine.

\section{Consent for publication}

Not applicable.

\section{Competing interests}

All authors declare that they have no competing interests.

Received: 20 February 2021 Accepted: 27 June 2021

Published online: 06 July 2021

\section{References}

1. Yang J, Liu X, Cao S, Dong X, Rao S, Cai K. Understanding esophageal cancer: the challenges and opportunities for the next decade. Front Oncol. 2020;10:1727 (Epub 2020/10/06)

2. Businello G, Parente P, Mastracci L, Pennelli G, Traverso G, Milione M, et al. The pathologic and molecular landscape of esophageal squamous cell carcinogenesis. Cancers (Basel). 2020;12(8):2160 (Epub 2020/08/08).

3. Zeng H, Zheng R, Guo Y, Zhang S, Zou X, Wang N, et al. Cancer survival in China, 2003-2005: a population-based study. Int J Cancer. 2015;136(8):1921-30 (Epub 2014/09/23).

4. Lin DC, Wang MR, Koeffler HP. Genomic and epigenomic aberrations in esophageal squamous cell carcinoma and implications for patients. Gastroenterology. 2018;154(2):374-89 (Epub 2017/08/02).

5. Chen W, Zheng R, Baade PD, Zhang S, Zeng H, Bray F, et al. Cancer statistics in China, 2015. CA Cancer J Clin. 2016;66(2):115-32 (Epub 2016/01/26)
6. Zheng RS, Sun KX, Zhang SW, Zeng HM, Zou XN, Chen R, et al. Report of cancer epidemiology in China, 2015. Zhonghua Zhong Liu Za Zhi. 2019:41(1):19-28.

7. Reichenbach ZW, Murray MG, Saxena R, Farkas D, Karassik EG, Klochkova A, et al. Clinical and translational advances in esophageal squamous cell carcinoma. Adv Cancer Res. 2019;144:95-135 (Epub 2019/07/28).

8. Liu Y, Xiong Z, Beasley A, D'Amico T, Chen XL. Personalized and targeted therapy of esophageal squamous cell carcinoma: an update. Ann NY Acad Sci. 2016;1381(1):66-73 (Epub 2016/10/28).

9. Ajani JA, D'Amico TA, Bentrem DJ, Chao J, Corvera C, Das P, et al. Esophageal and esophagogastric junction cancers, version 2.2019, NCCN clinical practice guidelines in oncology. J Natl Comp Cancer Netw. 2019;17(7):855-83.

10. Tian S, Quan H, Xie C, Guo H, Lu F, Xu Y, et al. YN968D1 is a novel and selective inhibitor of vascular endothelial growth factor receptor-2 tyrosine kinase with potent activity in vitro and in vivo. Cancer Sci. 2011;102(7):1374-80 (Epub 2011/03/30).

11. Scott LJ. Apatinib: a review in advanced gastric cancer and other advanced cancers. Drugs. 2018;78(7):747-58 (Epub 2018/04/18).

12. Aoyama T, Yoshikawa T. Targeted therapy: apatinib-new thirdline option for refractory gastric or GEJ cancer. Nat Rev Clin Oncol. 2016;13(5):268-70 (Epub 2016/5)

13. Yanwei L, Feng H, Ren P, Yue J, Zhang W, Tang P, et al. Safety and efficacy of apatinib monotherapy for unresectable, metastatic esophageal cancer: a single-arm, open-label, phase II study. Oncologist. 2020;25:e1464-72 (Epub 2020/04/29).

14. Lin YS, Wang C, Gao W, Cui RX, Liang J. Overwhelming rapid metabolic and structural response to apatinib in radioiodine refractory differentiated thyroid cancer. Oncotarget. 2017;8(26):42252-61 (Epub 2017/6/27).

15. Zhang J, Li A, Jiang Q, Zheng F, Zhu H. Efficacy and safety of apatinib treatment in platinum-resistant recurrent epithelial ovarian cancer: a real world study. Drug Des Dev Ther. 2019;13:3913-8 (Epub 2019/12/10).

16. Xu J, Zhang Y, Jia R, Yue C, Chang L, Liu R, et al. Anti-PD-1 Antibody SHR1210 combined with apatinib for advanced hepatocellular carcinoma, gastric, or esophagogastric junction cancer: an open-label, dose escalation and expansion study. Clin Cancer Res. 2019;25(2):515-23 (Epub 2018/10/24)

17. Li J, Jia Y, Gao Y, Chang Z, Han H, Yan J, et al. Clinical efficacy and survival analysis of apatinib combined with docetaxel in advanced esophageal cancer. Onco Targets Ther. 2019;12:2577-83 (Epub 2019/05/02).

18. Wei B, Wang Y, Wang J, Cai X, Xu L, Wu J, et al. Apatinib suppresses tumor progression and enhances cisplatin sensitivity in esophageal cancer via the Akt/beta-catenin pathway. Cancer Cell Int. 2020;20:198 (Epub 2020/06/10)

19. Liang $L$, Wen YX, Xia YY, Wang L, Fei JY, Jiang XD. Apatinib combined with docetaxel as a salvage treatment for metastatic esophageal squamous cancer: a case report. Onco Targets Ther. 2018;11:5821-6 (Epub 2018/10/03)

20. Berridge MJ. The endoplasmic reticulum: a multifunctional signaling organelle. Cell Calcium. 2002;32(5-6):235-49 (Epub 2002/11-12).

21. Gagnon E, Duclos S, Rondeau C, Chevet E, Cameron PH, Steele-Mortimer $\mathrm{O}$, et al. Endoplasmic reticulum-mediated phagocytosis is a mechanism of entry into macrophages. Cell. 2002:110(1):119-31 (Epub 2002/7/12).

22. Iurlaro R, Munoz-Pinedo C. Cell death induced by endoplasmic reticulum stress. FEBS J. 2016;283(14):2640-52 (Epub 2015/11/21).

23. Song S, Tan J, Miao Y, Li M, Zhang Q. Crosstalk of autophagy and apoptosis: involvement of the dual role of autophagy under ER stress. J Cell Physiol. 2017;232(11):2977-84 (Epub 2017/01/10).

24. Su Z, Yang Z, Xu Y, Chen Y, Yu Q. Apoptosis, autophagy, necroptosis, and cancer metastasis. Mol Cancer. 2015;14:48 (Epub 2015/03/10).

25. Lu $X$, Jin EJ, Cheng X, Feng S, Shang XY, Deng PN, et al. Opposing roles of TGF $\beta$ and BMP signaling in prostate cancer development. Genes Dev. 2017;31(23-24):2337-42 (Epub 2017/12/1).

26. National Research Council (US) Committee for the Update of the Guide for the Care and Use of Laboratory Animals. GUIDE for care and use of laboratory animals. Washington (DC): National Academies Press (US); 2011.

27. Blume-Jensen P, Hunter T. Oncogenic kinase signalling. Nature. 2001;411(6835):355-65 (Epub 2001/5/17).

28. Pene F, Claessens YE, Muller O, Viguié F, Mayeux P, Dreyfus F, et al. Role of the phosphatidylinositol 3-kinase/Akt and mTOR/P70S6-kinase pathways 
in the proliferation and apoptosis in multiple myeloma. Oncogene. 2002;21(43):6587-97 (Epub 2002/9/26).

29. Chen PM, Gombart ZJ, Chen JW. Chloroquine treatment of ARPE-19 cells leads to lysosome dilation and intracellular lipid accumulation: possible implications of lysosomal dysfunction in macular degeneration. Cell Biosci. 2011;1(1):10 (Epub 2011/06/30).

30. Hasanain M, Bhattacharjee A, Pandey P, Ashraf R, Singh N, Sharma S, et al. alpha-Solanine induces ROS-mediated autophagy through activation of endoplasmic reticulum stress and inhibition of Akt/mTOR pathway. Cell Death Dis. 2015;6:e1860 (Epub 2015/08/28).

31. Zhang HZ, Jin GF, Shen HB. Epidemiologic differences in esophageal cancer between Asian and Western populations. Chin J Cancer. 2002;31(6):281-6 (Epub 2002/6).

32. Pennathur A, Gibson MK, Jobe BA, Luketich JD. Oesophageal carcinoma. Lancet. 2013;381(9864):400-12.

33. Hirst J, Smithers BM, Gotley DC, Thomas J, Barbour A. Defining cure for esophageal cancer: analysis of actual 5-year survivors following esophagectomy. Ann Surg Oncol. 2011;18(6):1766-74 (Epub 2011/01/08)

34. McNamara MJ, Adelstein DJ. Current developments in the management of locally advanced esophageal cancer. Curr Oncol Rep. 2012;14(4):342-9 (Epub 2012/05/01).

35. Huang M, Huang B, Li G, Zeng S. Apatinib affect VEGF-mediated cell proliferation, migration, invasion via blocking VEGFR2/RAF/MEK/ERK and PI3K/AKT pathways in cholangiocarcinoma cell. BMC Gastroenterol. 2018;18(1):169 (Epub 2018/11/08).

36. Meng $X$, Wang $H$, Zhao J, Hu L, Zhi J, Wei S, et al. Apatinib inhibits cell proliferation and induces autophagy in human papillary thyroid carcinoma via the PI3K/Akt/mTOR signaling pathway. Front Oncol. 2020;10:217 (Epub 2020/03/29).
37. Xu Z, Hu C, Chen S, Zhang C, Yu J, Wang X, et al. Apatinib enhances chemosensitivity of gastric cancer to paclitaxel and 5 -fluorouracil. Cancer Manag Res. 2019;11:4905-15 (Epub 2019/06/20).

38. Li F, Zhu T, Cao B, Wang J, Liang L. Apatinib enhances antitumour activity of EGFR-TKIs in non-small cell lung cancer with EGFR-TKI resistance. Eur J Cancer. 2017;84:184-92 (Epub 2017/08/22)

39. Mizushima N. Autophagy: process and function. Genes Dev. 2007;21(22):2861-73 (Epub 2007/11/17).

40. Cheng YC, Chang JM, Chen CA, Chen HC. Autophagy modulates endoplasmic reticulum stress-induced cell death in podocytes: a protective role. Exp Biol Med (Maywood). 2015;240(4):467-76 (Epub 2014/10/18)

41. Wirawan E, Vande Walle L, Kersse K, Cornelis S, Claerhout S, Vanoverberghe I, et al. Caspase-mediated cleavage of Beclin-1 inactivates Beclin1 -induced autophagy and enhances apoptosis by promoting the release of proapoptotic factors from mitochondria. Cell Death Dis. 2010;1:e18 (Epub 2010/01/01).

42. Ogata M, Hino S, Saito A, Morikawa K, Kondo S, Kanemoto S, et al. Autophagy is activated for cell survival after endoplasmic reticulum stress. Mol Cell Biol. 2006;26(24):9220-31 (Epub 2006/10/13).

43. Ciechomska IA, Gabrusiewicz K, Szczepankiewicz AA, Kaminska B. Endoplasmic reticulum stress triggers autophagy in malignant glioma cells undergoing cyclosporine a-induced cell death. Oncogene. 2013;32(12):1518-29 (Epub 2012/05/15).

\section{Publisher's Note}

Springer Nature remains neutral with regard to jurisdictional claims in published maps and institutional affiliations.
Ready to submit your research? Choose BMC and benefit from:

- fast, convenient online submission

- thorough peer review by experienced researchers in your field

- rapid publication on acceptance

- support for research data, including large and complex data types

- gold Open Access which fosters wider collaboration and increased citations

- maximum visibility for your research: over $100 \mathrm{M}$ website views per year

At BMC, research is always in progress.

Learn more biomedcentral.com/submissions 